University of Warwick institutional repository: http://go.warwick.ac.uk/wrap This paper is made available online in accordance with publisher policies. Please scroll down to view the document itself. Please refer to the repository record for this item and our policy information available from the repository home page for further information.

To see the final version of this paper please visit the publisher's website. Access to the published version may require a subscription.

Author(s): S. Dixon and X. Jian Article Title: Eddy current generation enhancement using ferrite for electromagnetic acoustic transduction Year of publication: 2006 Link to published version: http://dx.doi.org/10.1063/1.2387125 Publisher statement: None 


\title{
Eddy current generation enhancement using ferrite for electromagnetic acoustic transduction
}

\author{
S. Dixon ${ }^{\text {a) }}$ and X. Jian \\ Department of Physics, University of Warwick, Coventry CV4 7AL, United Kingdom
}

(Received 26 July 2006; accepted 30 September 2006; published online 8 November 2006)

\begin{abstract}
Eddy currents are generated in an electrically conducting surface as a step in electromagnetic acoustic transduction (EAT). In eddy current testing, wire coils are often wound onto a ferrite core to increase the generated eddy current. With EAT, increased coil inductance is unacceptable as it leads to a reduction in the amplitude of a given frequency of eddy current from a limited voltage source, particularly where the current arises from capacitor discharge. The authors present a method for EAT where ferrite is used to increase the eddy current amplitude without significantly increasing coil inductance or changing the frequency content of the eddy current. (C) 2006 American Institute of Physics. [DOI: 10.1063/1.2387125]
\end{abstract}

The use of materials with a high relative magnetic permeability $\left(\mu_{r}\right)$ and low electrical conductivity $(\sigma)$ as transformer cores or cores of eddy current generating coils is well known and should not be confused with the approach that we describe here. A ferrimagnetic material ${ }^{1}$ such as ferrite will greatly increase the inductance of a coil due to its high relative magnetic permeability between 10 and $10^{3}$. Ferrite is composed of oxides of iron combined with one or more of the transition metals such that it has low electrical conductivity. Consequently, eddy currents are not generated in the ferrite by a current through a nearby coil. Generating an eddy current in the core of a coil would lead to a reduction in the inductance of the coil and a reduction in the eddy current that could be generated in the target sample derived from a fixed voltage source at a particular frequency of current discharge. $^{2}$

In the case of electromagnetic acoustic transduction an eddy current is generated in the electromagnetic skin depth of a sample. This eddy current interacts with the coil in the current itself to produce a repulsive force normal to the sample surface, generating an ultrasonic wave. ${ }^{2-4}$ In addition, a static magnetic field is often present, orientated perpendicularly to the direction of the eddy current, leading to a Lorentz force on the electrons which transfer momentum to the atoms and hence generate an ultrasonic wave. ${ }^{3,4}$ In the case of an eddy current testing, ${ }^{5}$ an eddy current is generated by a time varying current through a coil in proximity to the sample surface. The magnetic field from the eddy current is detected by a magnetic sensor, which detects a change in the eddy current's magnetic field due to either changes in the electromagnetic properties of the sample or a defect in the skin depth.

When one uses an electromagentic acoustic transducer (EMAT) to generate ultrasound in a sample, a pulse of current is passed through the coil. This pulse of current is typically obtained by capacitor discharge, and one can reasonably represent the circuit as a dishcharge capacitor in series with an inductor and a smaller capacitor that are themselves in parallel. ${ }^{1}$ In what follows, we limit ourselves to discussing the case of an EMAT that does not contain a permanent magnet, where the ultrasonic generation arises from the in-

\footnotetext{
${ }^{a)}$ Electronic mail: s.m.dixon@ warwick.ac.uk
}

teraction between the current in the coil and the eddy current in the sample surface. This interaction gives rise to a force that is predominantly normal to the surface, but there are also some smaller in-plane force components. ${ }^{5}$

An EMAT detector that predominantly has sensitivity to in-plane displacements of the sample surface was used to detect and measure a Rayleigh wave generated by an EMAT coil, as shown schematically in Fig. 1. As a detector, the EMAT acts as a velocity sensor. ${ }^{6}$ If the shape of the pulse remains approximately the same though, one can relate any detected change in amplitude of the EMAT signal directly to a change in the amplitude of the ultrasonic wave. The detection EMAT is a linear coil with a coil width of $1 \mathrm{~mm}$, and the generation coil is a flat spiral coil of ten turns of a $0.25 \mathrm{~mm}$ diameter enamel insulated copper wire with its center approximately $170 \mathrm{~mm}$ from the center of the detection coil. The current is pulsed through the generation coil by a capacitor discharge where the capacitor is charged to a fixed voltage, and the discharge is switched by solid state electronic devices.

The resultant time-displacement plots or A scans are shown in Fig. 2 for the cases where no backing material is used and when a sample of ferrite is used as the backing material. The standoff of the coil from the sample surface is $0.1 \mathrm{~mm}$ in each case, and where the ferrite material is used it is placed in direct contact with the coil.

The presence of the ferrite has very little effect on the shape and hence frequency content of the Rayleigh wave, but the amplitude is increased by a factor of $1.5 \pm 0.05$. The same increase in amplitude is observed for bulk waves generated using the approach with the spiral generation coil for a range of standoffs of up to $1.5 \mathrm{~mm}$.

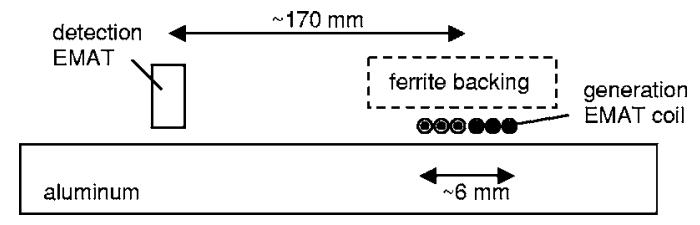

FIG. 1. Schematic diagram of the experimental setup used to measure the ultrasonic displacement produced by the compression wave that is generated by the EMAT. 


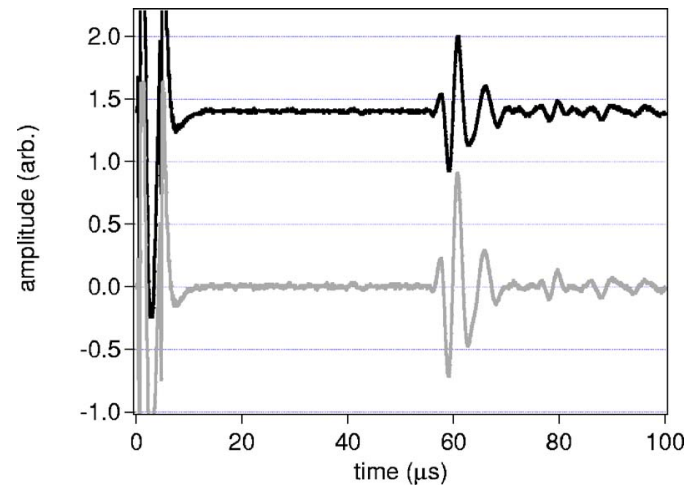

FIG. 2. In both of the above traces the Rayleigh wave is the feature at approximately $58 \mu \mathrm{s}$, and the smaller feature at approximately $78 \mu \mathrm{s}$ is a Rayleigh wave reflection from the edge of the sample. The upper trace (black) shows the detected Rayleigh wave without any backing material present and the lower trace (gray) shows that with the ferrite backing in place.

The current through the coil is also monitored by measuring the voltage across a $0.02 \Omega$, noninductive resistor in series with the coil, and the plot of current against time during the capacitor discharge is shown in Fig. 3.

Previous work has shown that the current through the coil is directly related to the ultrasonic wave displacement generated by the EMAT. ${ }^{7}$ There is no discernable difference in the current through the coil in the presence of the ferrite backing material when the coil is in close proximity to the aluminum. Thus the ferrite has an insignificant effect on the current in the coil. If one examines the amplitude of the ultrasonic arrivals shown in Fig. 2, then it is clear that there is a strong enhancement in the generated amplitude of the ultrasonic wave. The key point is that the ferrite has significantly increased the eddy current in the coil without significantly changing the inductance of the coil, which would have limited the frequencies of eddy current or ultrasonic wave that we generate. Even when one performs measurements in the extreme cases of the coil in proximity to an aluminum sample alone and then in proximity to a ferrite sample alone, the difference between the current pulse widths and amplitudes is less than 3\%. Modeling also shows that the exact level of increase in the eddy current generated by the coil is dependent on the standoff of the coil from the sample, the separation of the coil and ferrite, the type of coil, and the geometry of the sample. In all cases, however, there is a

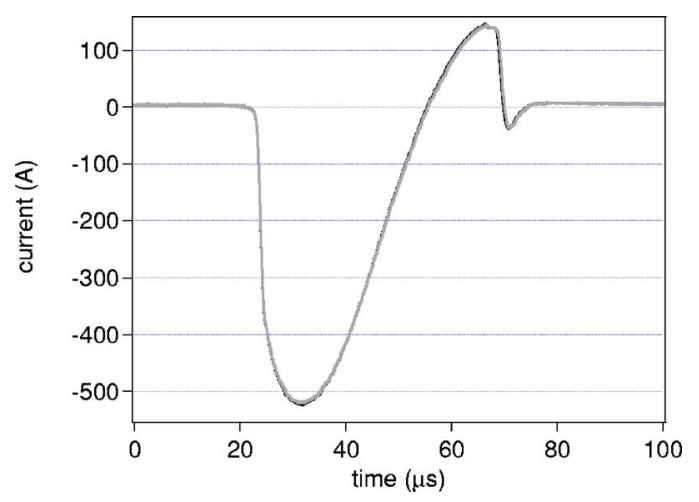

FIG. 3. The above overlapping traces show the measured current through the coil in close proximity to an aluminum sample with no backing material present (black) and with the ferrite backing in place (gray). The traces are almost identical, and it is not possible to differentiate them from each other.

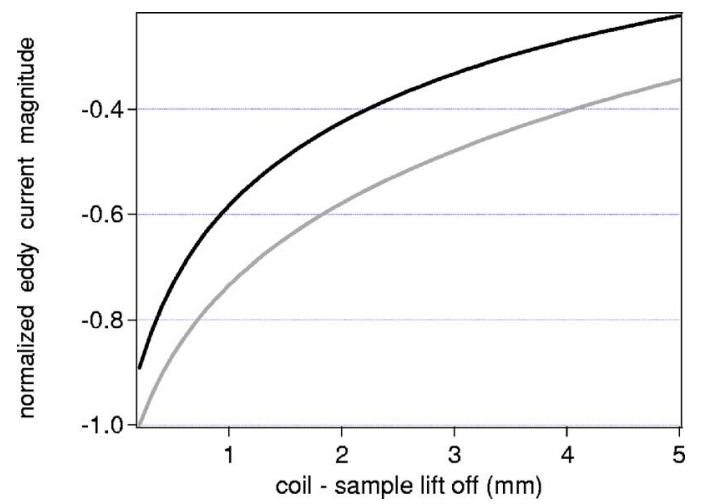

FIG. 4. Calculated normalized maximum eddy current in the aluminum sample for a $300 \mathrm{kHz}$ component of current at a depth of $0.05 \mathrm{~mm}$ below the sample surface for a $10 \mathrm{~mm}$ diameter five-turn flat spiral coil with no backing material present (black) and with the ferrite backing in place (gray).

significant enhancement in the eddy current amplitude, typically by a factor between $130 \%$ and $400 \%$.

The effect of the ferrite backing layer on a flat spiral coil and the resultant magnetic field flux density in the sample skin depth has been modeled analytically and computed using finite element methods for the case of the spiral coil. This modeling was actually performed prior to the experiments and directed us towards the experimental measurements. The theoretical predictions for the maximum eddy current at $300 \mathrm{kHz}$ as a function of standoff, $0.05 \mathrm{~mm}$ below the sample surface for the case of a $10 \mathrm{~mm}$ diameter coil of five turns of $0.1 \mathrm{~mm}$ diameter copper wire with a coil-ferrite separation of $0.2 \mathrm{~mm}$, are shown in Fig. 4. These data were normalized to the maximum predicted value obtained in the case with a ferrite backing present behind the coil.

The cause of the enhancement of the eddy current is the magnetization of the ferrite behind the coil. When current is pulsed through the coil, provided the ferrite material is one which can respond quickly enough to the changing magnetic field, the ferrite becomes magnetized. A contour plot of the magnetic flux density is shown in Fig. 5 at a time during the current pulse that corresponds to the maximum magnetic flux density in the sample. Figure 5 has been calculated for a spiral coil of four turns with a coil diameter of $6 \mathrm{~mm}$ for the cases with no ferrite present (left) and with a ferrite backing present (right). Comparing these two cases, outside the aluminum sample and behind the coil, the magnetic flux density profile is dramatically different due to the high relative permeability of the ferrite. Inside the aluminum sample the magnetic flux density profile is fairly similar, the main difference being the amplitude of the magnetic flux density, which is higher for the case with the ferrite backing.

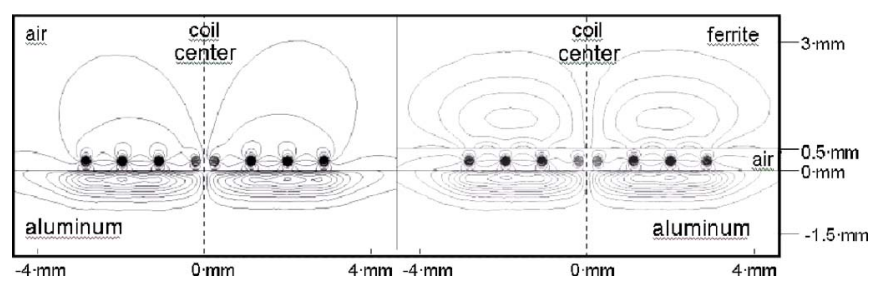

FIG. 5. Contour plots of in-plane magnetic flux density, calculated for a flat spiral coil of four turns with a coil diameter of $6 \mathrm{~mm}$ for the cases with no ferrite present (left) and with a ferrite backing present (right). Note that the contours can appear discontinuous at the boundaries as the contours are for magnetic flux density and not for magnetic flux lines. 
In conclusion we present a generic technique for increasing the eddy current that one can generate in a sample without significantly changing the electrical inductance of the coil and hence not significantly reducing the amplitude of a particular frequency component of that eddy current. This has been done by placing a coil of wire in proximity to the ferrite material and not wrapping the coil around the ferrite material, which would make it act as a transformer core. More specifically, we have shown that the approach we describe can be used to increase the efficiency of an EMAT for the generation of ultrasonic waves in metallic samples. In some instances, a ferrite backing material behind a coil will produce a larger amplitude ultrasonic wave than a favorably orientated, high field $\mathrm{NdFeB}$ permanent magnet backing material will produce. ${ }^{7}$ The type of EMAT generation that we have described here is actually due to the Lorentz force, but the enhancement of the dynamic magnetic field obtained with a ferrite will also increase the efficiency of EMATs designed to generate ultrasound via a magnetoelastic mechanism such as magnetostriction. There will be many other applications where this approach can be used to increase the amplitude of eddy current generation without significant loss of bandwidth, particularly at frequencies above $50 \mathrm{kHz}$ up to several megahertz.

${ }^{1}$ C. Kittel, Introduction to Solid State Physics (Wiley, New York, 2005), p. 336.

${ }^{2}$ X. Jian, S. Dixon, R. S. Edwards, and J. Reed, J. Acoust. Soc. Am. 119, 2693 (2006).

${ }^{3}$ H. M. Frost, in Physical Acoustics XIV, edited by W. P. Mason and R. N. Thurston (Academic, New York, 1979), pp. 179-275.

${ }^{4}$ R. B. Thompson, in Physical Acoustics XVIII, edited by R. N. Thurston and A. D. Pierce (Academic, New York, 1990), pp. 157-200.

${ }^{5}$ A. Sophian, G. Y. Tian, D. Taylor, and J. Rudlin, Insight 43, 302 (2001).

${ }^{6}$ K. Kawashima, J. Acoust. Soc. Am. 60, 1089 (1976).

${ }^{7}$ S. Dixon and S. B. Palmer, Ultrasonics 42, 1129 (2004). 